Revue internationale de l'économie sociale

Recma

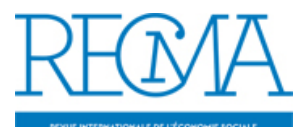

\title{
En bref
}

\section{La rédaction}

Numéro 334, octobre 2014

URI : https://id.erudit.org/iderudit/1027271ar

DOI : https://doi.org/10.7202/1027271ar

Aller au sommaire du numéro

\section{Éditeur(s)}

Association Recma

\section{ISSN}

1626-1682 (imprimé)

2261-2599 (numérique)

Découvrir la revue

Citer ce document

La rédaction (2014). En bref. Revue internationale de l'économie sociale, (334), 16-26. https://doi.org/10.7202/1027271ar d'utilisation que vous pouvez consulter en ligne.

https://apropos.erudit.org/fr/usagers/politique-dutilisation/ 


\section{EN BREF}

\section{L’économie sociale}

\section{Première réunion du groupe pilote international de $\mathbf{l}^{\prime} \mathrm{ESS}$}

Le groupe pilote international de l'ESS, créé en novembre 2013 et composé d'Etats, d'institutions internationales, du Forum international des dirigeants de l'ESS et d'organisations alliées des mondes coopératifs, mutualistes, associatifs et des fondations, s'est réuni pour la première fois le 22 septembre dernier au siège de l'ONU, en marge de l'assemblée générale de cette organisation. L'association des Rencontres du Mont-Blanc, à l'origine de la création de la plateforme, a organisé cette réunion, marquée par deux sujets principaux: "le développement de l'ESS dans le monde, et particulièrement les politiques publiques en direction de l'ESS (législations...); le soutien à la création et au développement d'entreprises de l'ESS dans le monde, notamment à travers la question des financements $"$. Pour celles et ceux qui souhaiteraient revivre ce moment, une vidéo de l'événement est disponible (en anglais) sur la WebTV de l'ONU: webtv.un.org.

\section{Une étude européenne sur l'égalité femmes-hommes dans l'ESS et l'économie classique} Six partenaires issus d'autant de pays européens (dont, pour la France, la fondation Agir contre l'exclusion) se sont réunis en un consortium chargé de mener le projet européen "Gender Balance Power Map: Co-inspiration between social and classic enterprises on access to decision making ". Ce dernier repose sur l'hypothèse que les entreprises classiques et celles de l'économie sociale et solidaire présentent des points communs, mais aussi des différences quant à la question de l'égalité homme-femme, et plus précisément de l'accès des femmes aux responsabilités. Lancé par le think tank
Pour la solidarité, il est financé dans le cadre du programme européen Progress-Gender Equality. Il se déclinera en dix-huit ateliers nationaux avec des entreprises classiques et sociales, plusieurs séminaires et une conférence finale (prévue en mai 2016) et donnera lieu à la production de six études nationales, une étude comparative européenne et un guide européen des bonnes pratiques, assorti de recommandations.

\section{Action gouvernementale en faveur de l'ES en outre-mer}

Le 30 septembre dernier, François Rebsamen, ministre de l'Emploi, Emmanuel Macron, ministre de l'Economie, Carole Delga, secrétaire d'Etat chargée de l'ESS, et Pierre-René Lemas, directeur général de la Caisse des dépôts, ont signé la convention «Agir pour l'emploi et la création d'activités, 20142017 ». Cette dernière se donne quatre buts: "une meilleure adaptation des outils de financement à la création d'entreprises aux publics et aux structures; le soutien à l'accompagnement des créateurs de microentreprise et de TPE; la préparation de la France au défi démographique de départ à la retraite des dirigeants d'entreprise d'ici à 2020; le développement de l'économie sociale et solidaire sur tout le territoire». Elle prévoit pour cela un engagement de l'Etat à hauteur de 80,6 millions d'euros et de la Caisse des dépôts à hauteur de 45 millions.

A cette occasion, George Pau-Langevin, ministre des Outre-mer, a signé le protocole de partenariat entre l'Etat et la Caisse des dépôts déclinant les actions de la convention en Outre-mer. Celui-ci se concentre sur deux objectifs: le soutien à l'embauche d'un premier salarié dans les TPE et le soutien à l'ESS, qui "pourra prendre la forme d'une aide financière à la création, à la reprise ou au développement des structures existantes, en privilégiant particulièrement l'aide à 
l'investissement ou à la structuration et la professionnalisation des réseaux de l'ESS et des entités qui les composent. Ce soutien pourra également prendre la forme d'une participation au financement d'actions retenues dans le cadre d'appels à projets "Pôles territoriaux de coopération économique" (PTCE), afind'encourager l'émergence de projets menés dans les territoires ultramarins ». Ces deux objectifs seront soutenus par le ministère à hauteur de 2,5 millions d'euros chacun, avec comme perspective la création de 4000 emplois sur quatre ans.

\section{Journée nationale des PTCE}

La II Journée nationale des pôles territoriaux de coopération économique (PTCE) s'est tenue le 19 septembre au ministère de l'Economie et des Finances, à Paris. Elle était organisée par le collectif inter-réseaux PTCE, composé du Labo de l'ESS, du Réseau des collectivités territoriales pour l'économie solidaire (RTES), du Conseil national des chambres régionales de l'économie sociale (CNcres), du Coorace et du Mouvement pour l'économie solidaire.

Le Labo de l'ESS s'est félicité de son succès, avançant le chiffre de quatre cents personnes réunies à cette occasion. Celles-ci ont pu entendre Sylvia Pinel, ministre du Logement, de l'Egalité des territoires et de la Ruralité, ouvrir cette journée et ont pu assister, pour la clôture, à la signature par Carole Delga, secrétaire d'Etat chargée du Commerce, de l'Artisanat, de la Consommation et de l'Economie sociale et solidaire, de la Charte de la démarche collective des PTCE.

Point d'orgue de la journée, cette dernière vise à formaliser la démarche collective. Son objet est de "faire reconnaître l'importance des processus de coopérations économiques territoriales initiés par les acteurs et les entreprises de l'ESS porteurs de nouveaux modèles économiques sources de richesse économique, sociale et environnementale; [de] faire vivre ces dynamiques de coopération dans les territoires; [de] valoriser leur utilitésociale; [d'] inciter à la mise en cohérence des politiques territoriales ». Elle affirme également les valeurs sur lesquelles les PTCE doivent s'appuyer, parmi lesquelles la valorisation du patrimoine local, la co-construction, la solidarité et la recherche d'innovations sociales.

Carole Delga, interrogée par le journal La Croix, a fait le lien avec la toute récente loi sur l'ESS: "Promulguée le 31 juillet dernier, [celle-ci] agit sur l'ensemble des domaines de développement de l'économie sociale et solidaire (ESS): la reconnaissance de cette forme d'économie, ses acteurs, son financement. Le développementéconomique des territoires en est un des axes forts. Il passe par les PTCE, mais aussi par la reconnaissance des chambres régionales de l'ESS (Cress) et le renforcement des sociétés coopératives d'intérêt collectif(Scic) - qui sont une structure juridique pleine d'avenir - et des coopératives d'activité et d'emploi (CAE).»

\section{Le rapport du CNCIIS}

\section{sur l'investissement à impact social}

En juin 2013, a été constitué un groupe de travail international sur l'investissement à impact social par les Etats du G8. C'est dans ce cadre que s'inscrivent, en France, les travaux du Comité national consultatif sur l'investissement à impact social (CNCIIS), présidé par Hugues Sibille. Le CNCIIS a travaillé pendant un an, en parallèle des débats sur la loi-cadre ESS votée en juillet dernier, à l'élaboration d'un rapport, qui a été remis le 29 septembre dernier à Carole Delga, secrétaire d'Etat chargée de l'ESS.

Grâce à la participation de "vingt-neuf personnalités issues du monde de la banque, du capital investissement, de l'entrepreneuriat social, d'agences publiques, de milieux académiques et d'organisations internationales, ainsi que de représentants de l'Etat, de BPIFrance et de la Caisse des dépôts", celui-ci présente des propositions concrètes organisées selon six axes: "améliorer l'adéquation entre l'offre de financement et les besoins des entreprises à impact social; augmenter les ressources financières 
disponibles; favoriser l'émergence d'entreprises à impact social; développer l'investissement orienté sur des missions sociales définies, par les fonds et les fondations; stimuler l'investissement à impact social pour le développement international; mettre en place un cadre de référence pour mesurer l'impact social.

Le rapport dresse un état des lieux $d u$ modèle français de la finance solidaire, organisé notamment autour de l'existence d'une épargne salariale solidaire ou d'investisseurs publics spécialisés dans le champ solidaire, ce qui permettra à la France de se positionner de manière efficace et crédible dans les discussions internationales sur la finance sociale.

Parmi ces propositions, figurent notamment des pistes pour amplifier l'épargne salariale solidaire. Le rapport préconise également de décliner les vecteurs d'investissement solidaires au sein d'autres supports d'épargne que l'épargne salariale, et notamment au sein de l'assurance-vie."

A cette occasion, Carole Delga a déclaré que «la recherche d'un impact social à court terme par les investisseurs solidaires n'éclipsera pas ce qui fonde le modèle français de l'investissement à impact social: le maintien à long terme des solidarités construites autour à la fois de l'entreprise solidaire et des mécanismes de solidarité nationale, mais aussi le rôle essentiel des services publics ».

\section{L'Apec investit le champ de l'ESS}

L'Association pour l'emploi des cadres (Apec) multiplie les actions en direction de l'ESS. De mai à décembre 2014, elle organisera plus de quarante ateliers dans vingtquatre villes de France avec pour objectif de conseiller les employeurs de l'ESS, de promouvoir auprès du public les emplois dans l'ESS et d'informer sur la création et la reprise d'entreprise dans le secteur. Marie-José Batlle, responsable du développement des activités institutionnelles et partenariales au sein de l'Apec, a eu l'occasion de vanter le succès de cette opération auprès du Labo de l'ESS, auquel elle a déclaré: "Nous souhaitons être au plus proche de la réalité des territoires en livrant des informations précises et très pratico- pratiques, en communiquant des listes de relais locaux. Le partage d'expériences est également essentiel, et nous invitons de nombreux acteurs de l'ESS à témoigner. [...] Nous sommes agréablement surpris de voir combien notre public s'intéresse à l'ESS, que ce soit des cadres en pleine réflexion sur leur avenir professionnel, des jeunes diplômés ou des porteurs de projet. »

Cela fait maintenant deux ans que l'Apec a commencé à investir ce champ de l'économie, depuis qu'elle s'est rapprochée du CNcres avec lequel elle avait publié une étude commune (fin 2012) sur le thème " Les cadres de l'ESS ».

\section{Propositions de l'Udes aux Assises de l'apprentissage}

Le 19 septembre dernier, ont eu lieu, sous le haut patronage de la présidence de la République, les Assises de l'apprentissage. A cette occasion, l'Union des employeurs de l'économie sociale (Udes) a présenté les propositions suivantes:

- autoriser un apprenti à commencer son contrat en cours d'année. Il pourra ainsi saisir une opportunité d'embauche dans une entreprise lorsque celle-ci se présentera et faire son entrée en centre de formation tout au long de l'année;

- octroyer systématiquement, sur les fonds régionaux, une aide à l'obtention du permis pour un apprenti dans les secteurs d'activité où les déplacements avec un véhicule personnel sont une condition requise pour l'obtention de l'emploi concerné (services à domicile);

- généraliser la mise en place de task force ("groupe de travail ») territoriales dédiées à l'alternance en assurant la mobilisation de chaque opérateur de l'emploi (missions locales, Pôle emploi, Cap emploi...);

- introduire un module relatif aux spécificités de la formation par apprentissage 
et aux pédagogies de l'alternance dans le cursus des enseignants au sein des écoles supérieures du professorat et de l'éducation; - instaurer dans le parcours scolaire des modules sur les modes de fonctionnement de l'entreprise, pour éviter un décalage trop brutal entre le vécu à l'école et la réalité du monde du travail et ainsi assurer le succès de l'intégration professionnelle du jeune;

- augmenter les moyens financiers de l'Etat et des régions en faveur de l'aide au logement et de l'aide à la mobilité pour les apprentis. Il s'agit de répondre aux besoins spécifiques des jeunes en alternance.

Alain Cordès, président de l'Udes, a déclaré que "le recours au contrat d'apprentissage contribue à renouveler les équipes et à diversifier les canaux de recrutement classiques. La présence des apprentis permet d'organiser la transmission des savoirs et de valoriser ainsi les salariés en seconde partie de carrière. Pour les jeunes, démarrer leur vie active dansl'économie sociale et solidaire leur permet d'intégrer des formes d'entreprises qui privilégient le service rendu, tout en étant de véritables acteurs économiques».

\section{La coopération}

\section{Desjardins au sommet}

Le mouvement Desjardins, premier groupe financier coopératif du Canada et quatrième mondial, a déclaré avoir connu au deuxième trimestre 2014 le meilleur trimestre de son histoire. Dans un communiqué, il a donnéles précisions suivantes: "[Le groupe] connaît une solide croissance dans tous ses secteurs d'affaires et enregistre des revenus d'exploitation de 3157 millions de dollars canadiens, en hausse de 7,4\% par rapport à ceux du trimestre correspondant en 2013. La croissance des affaires liées aux activités d'assurance a généré une augmentation de 159 millions de dollars canadiens, soit de $11,9 \%$, des primes nettes, qui atteignent 1499 millions de dollars canadiens. Cette performance lui permet d'afficher des excédents avant ristournes aux membres de 446 millions de dollars canadiens, comparativement aux 387 millions de dollars canadiens au même trimestre en 2013. » Monique Leroux, présidente du mouvement Desjardins, s'est félicitée de la pertinence des choix stratégiques du groupe et d'" une offre de service bien adaptée aux besoins de nos membres et de nos clients ». Par ailleurs, au moment de l'annonce de ces résultats florissants, on apprenait que le groupe Desjardins se trouvait placé à la deuxième place du classement Bloomberg des banques les plus solides du monde. Il est à noter qu'il partageait cette place avec une autre banque coopérative: la japonaise Norinchukin Bank.

Ces bonnes nouvelles sont arrivées à point, juste avant la tenue du $\mathrm{II}^{\mathrm{e}}$ Sommet international des coopératives, co-organisé par Desjardins et l'Alliance coopérative internationale (ACI).

\section{II $^{\mathrm{e}}$ Sommet international des coopératives}

Du 6 au 10 octobre dernier, à Québec (Canada), s'est tenue la deuxième édition du Sommet international des coopératives, sous l'égide de l'Alliance coopérative internationale (ACI) et du mouvement Desjardins. Réunissant près de 3000 personnes venues de 93 pays, le sommet, dont un compte rendu sera publié dans le prochain numéro de la revue, a été sans aucun doute un succès. Y ont été célébrés les 2,6 millions de coopératives dans le monde, leur milliard de membres et les 250 millions d'emplois qu'elles représentent ( $12 \%$ de l'emploi total des pays du G20 et 3000 milliards de dollars américains de revenus annuels).

A cette occasion, l'ACI a dévoilé son rapport annuel sur les coopératives, le World Co-operative Monitor, coproduit avec le European Research Institute on Cooperatives and Social Enterprises (Euricse). Selon celuici, les trois cents plus grandes coopératives mondiales ont des revenus de 2200 milliards de dollars américains, soit une croissance de $11,2 \%$ sur la dernière année. C’est une coopérative japonaise, Zenkyoren, qui 
domine le classement. L'intégralité du rapport est consultable, en anglais, depuis le site de l'ACI: ica.coop/fr.

\section{Les coopératives agricoles japonaises menacées par une réforme}

En juillet dernier, l'Alliance coopérative internationale (ACI) a publié un communiqué pour dénoncer une proposition de réforme qui menace les coopératives agricoles au Japon. Les explications données par l'ACI: "Cette nouvelle réforme propose la dissolution des coopératives agricoles polyvalentes, de même que la réduction du rôle du syndicat central des coopératives agricoles (JA-Zenchu) en le transformant en un groupe de réflexion pour la promotion de l'agriculture. La proposition de réforme diluerait également la propriété des membres, puisqu'elle insiste pour que plus de la moitié des membres des conseils des coopératives agricoles soient des producteurs agricoles certifiés ou des chefs d'entreprise de sociétés privées (qui n'ont pas besoin, ni les uns ni les autres, d'être membres). Actuellement, conformément aux principes mondiaux associés aux coopératives, la loi stipule que deux tiers des membres du conseil doivent être des fermiers membres. La proposition de réforme appelle également à modifier la structure de propriété des terres agricoles. »

Les acteurs du secteur s'organisent pour combattre ces mesures, et l'ACI a annoncé les soutenir par la création d'un "groupe de contact et d'étude " qui examinera l'évolution de la situation. Ce dernier est composé de la présidente de l'ACI, Dame Pauline Green, du président du Crédit coopératif, Jean-Louis Bancel, et, enfin, du représentant international des coopératives électriques rurales nationales des Etats-Unis, Martin Lowery. Dame Pauline Green a déclaré que "l'ensemble du mouvement coopératif mondial, propriété d'un milliard de citoyens du monde entier, se tiendra aux côtés de ses collègues coopérateurs japonais pour s'opposer au démantèlement du mouvement coopératif agricole japonais et à la réduction consécutive des droits de ses membres".

\section{Une nouvelle constitution \\ pour le Réseau mondial des jeunes coopérateurs}

Créé en 2003, le réseau Jeunesse de l'Alliance coopérative internationale a élaboré sa nouvelle constitution, dont le texte a été approuvé en juin dernier. Il a été créé pour aider les jeunes coopérateurs du monde entier à construire des réseaux et à partager expériences et idées et vise à fournir un environnement propice à leur apprentissage sur le mouvement coopératif, les impliquer par l'éducation et le soutien, leur permettre de s'engager et d'accroître la visibilité des questions sur la jeunesse, autant de missions réaffirmées dans ce nouveau texte, consultable en anglais sur le site de l'ACI.

\section{Maroc: une loi et un recensement}

Le 16 juillet dernier, le parlement marocain a enfin voté le projet de loi relatif aux coopératives que le conseil de gouvernement avait adopté depuis 2012. Ce texte vise à normaliser le droit marocain des coopératives au regard des pratiques internationales et à simplifier la procédure de constitution de ce type de structure. Il prévoit la suppression de l'agrément préalable à l'exercice et établit des registres, nationaux et locaux, d'immatriculation des coopératives. Enfin, cette nouvelle loi instaure une procédure de règlement des litiges pilotée par les unions de coopératives ou la Fédération nationale des coopératives.

Ce vote du parlement intervient alors que l'Office du développement et de la coopération (Odco) opère un recensement général des coopératives du pays, à propos desquelles les chiffres précis manquent, le dernier recensement de ce type datant de 1998. Le nombre de structures ayant demandé un agrément dépassait les 12600 fin avril 2014, mais le recensement devrait préciser combien sont encore en activité. 
Sur ce nombre, $66 \%$ sont des coopératives agricoles, $15 \%$ d'artisanat et $9 \%$ d'habitat. L'Odco estime à moins de $2 \%$ la contribution du secteur coopératif au PIB, pour un taux de pénétration de $4 \%$. Le prochain rapport de cet organisme, à la suite du recensement, est attendu pour la fin de l'année.

Alors que les institutions du pays marquent par là une volonté réelle de consolidation et de développement du secteur, le British council et la Banque mondiale ont annoncé qu'ils allouaient 300000 dollars à l'accompagnement et au financement d'entreprises de l'entrepreneuriat social au Maroc.

\section{Sept coopératives agricoles françaises dans le top 20 européen}

La coopération agricole se porte mieux en France que dans le reste de l'Europe. C'est ce qui semble ressortir du classement des vingt premières coopératives agricoles européennes, établi par le cabinet d'audit et de conseil PwC, où figurent sept coopératives françaises, pays de ce fait le mieux représenté. Entre 2008 et 2013, la croissance des dix premières coopératives françaises a atteint $7,4 \%$ par an, soit $0,4 \%$ de plus que les cinquante premières coopératives mondiales. Cependant, comme le rapporte le site Terre-net.fr, Philippe Mangin, président de Coop de France (CoopFr), souligne que les bons résultats des plus grosses coopératives masquent une baisse de l'activité de 2,6\% de l'ensemble du secteur depuis 2012 : «Il déplore que le carnet de commandes des industries agroalimentaires étrangères soit plein pour des années, mais pas ceux des Françaises." Le contexte est donc globalement difficile pour les coopératives agricoles. Pour $\mathrm{PwC}$, "la taille est l'un des six principaux leviers qu'elles actionnent pour relever ce défi, avec l'internationalisation de leurs activités, l'intégration verticale en amont et en aval de leur portefeuille d'activités par acquisitions ou partenariats, le développement de marques fortes, l'innovation et le développement defilières inclusives avec d'autres acteurs privés et publics». Terre-net.fr précise que, si la France est bien placée pour l'export de certains produit «à forte valeur», elle peine en revanche à se positionner sur le marché des produits de "premier prix ».

\section{Les mutuelles}

\section{Les propositions mutualistes contre} la hausse des inégalités sanitaires

Longtemps vantée pour la qualité de son système de protection sociale, la France connaît aujourd'hui une dégradation inédite de l'accès aux soins. Selon une étude de l'Institut de recherche et de documentation en économie de la santé (Irdes), en 2012, près d'un Français sur trois a différé des soins médicaux, quand il n'y a pas simplement renoncé, pour des raisons financières. Parmi ceux-ci, figurent majoritairement des personnes n'ayant pas les moyens de souscrire à une complémentaire santé, d'autant que les tarifs des mutuelles ont augmenté ces dernières années, en raison d'une taxation de plus en plus lourde. Si préoccupante qu'elle soit, la fracture sanitaire n'est pas inéluctable. Pour y remédier, la Mutualité française a émis diverses pistes énoncées par son président, Etienne Caniard, à l'occasion d'une conférence de presse tenue le 30 septembre 2014. Parmi les principales propositions: baisse des taxes pesant sur les contrats santé; généralisation du tiers payant déjà mis en place par les mutuelles; création d'un organisme indépendant pour piloter les données de santé; rénovation du cadre des négociations conventionnelles avec les professions de santé; lutte contre les dépassements d'honoraires; renforcement de l'identité mutualiste, de ses valeurs et de sa gouvernance... Ce qui apparaît comme une urgence à la veille de la transposition de la directive Solvabilité II; refonte du Code de la mutualité.

\section{La LMDE dans la tourmente}

La précarité sanitaire touche particulièrement les étudiants. Or, selon une enquête de l'Observatoire de la vie étudiante (OVE) 
réalisée en 2013, le renoncement aux soins pour des raisons financières serait encore plus important chez les adhérents d'une mutuelle étudiante que chez les étudiants couverts par une mutuelle parentale, une mutuelle non étudiante ou un contrat d'assurance. En cause: la mauvaise qualité des services et des remboursements trop tardifs. Ces critiques corroborent les résultats de l'enquête de l'UFC-Que Choisir sur les mutuelles étudiantes publiée en septembre 2012, ainsi que le rapport 2013 de la Cour des comptes fustigeant leur gestion déficiente. Particulièrement visée, La Mutuelle des étudiants (LMDE) a été placée, en juillet dernier, sous administration provisoire par l'Autorité de contrôle prudentiel et de résolution (ACPR), avec un plan d'adossement - en panne à ce jour - à la MGEN, soutien historique de la mutuelle étudiante.

Les mésaventures de la LMDE réactivent désagréablement le souvenir du «scandale de la Mnef ", son ancêtre en ligne directe, qui avait animé l'actualité sociale en 1998-1999. Elles questionnent le particularisme du régime étudiant établi en 1948, par lequel les mutuelles bénéficient, comme les mutuelles de fonctionnaires, d'une délégation de service public. Jugé dispendieux par la Cour des comptes, le régime étudiant pourrait bien être transféré vers les caisses d'assurance maladie pour la gestion de la part obligatoire. Autrement dit, non seulement les déboires de la LMDE n'arrangeront pas forcément les affaires de Emevia, le réseau concurrent constitué de onze mutuelles régionales, mais ils risquent aussi de servir d'argument à tous ceux qui souhaiteraient, par principe, la suppression de la délégation de gestion aux mutuelles de fonctionnaires.

\section{Les mutuelles communales:}

\section{idée neuve ou retour au XIX ${ }^{\mathrm{e}}$ siècle?}

Afin de venir en aide aux personnes qui n'ont pas les moyens d'adhérer à une complémentaire santé, plusieurs municipalités ont pris l'initiative de créer une mutuelle communale, en négociant pour leurs habitants des contrats avantageux auprès d'autres mutuelles sur la base d'un cahier des charges exigeant. Voilà qui ne manque pas d'interpeller, à une époque où le mouvement mutualiste n'imagine pas d'autre issue que les fusions pour faire face à l'emprise conjuguée du marché et des contraintes prudentielles européennes. Des communes aux noms fleurant bon le terroir français comme Caumont-sur-Durance ou La-Bastide-desJourdans se sont lancées dans cette aventure solidaire largement relayée par les médias, qui oublient généralement la filiation historique de ces pratiques pour les présenter comme " une idée neuve ». Dès la première moitié du XIX ${ }^{e}$ siècle, en effet, des sociétés de secours mutuels ont été organisées à l'échelle d'une commune ou d'un quartier, pour la prise en charge notamment de l'assurance maladie. Elles ont été encouragées sous une forme patronnée par le Second Empire, puis sous une forme libérée par la Troisième République, avant la mise en place des régimes publics de protection sociale (Assurances sociales, Sécurité sociale) qui ont entraîné leur recul au profit des mutuelles professionnelles. Certes, il faut faire une lecture optimiste et réconfortante de la floraison des initiatives solidaires, mais elles n'en témoignent pas moins d'une régression de la capacité de l'Etat à assurer une protection sociale élémentaire. Par ailleurs, reste à définir la gouvernance de ces mutuelles communales, qui semblent moins relever d'une démarche bottom up que du dynamisme des élus. Affaire à suivre...

\section{Mutuelles-assurances}

Le statut juridique des sociétés de groupe d'assurance mutuelle (SGAM), créé en 2002, a été adopté dès 2003 par la société de réassurance mutuelle Covéa, qui réunit aujourd'hui la GMF, la Maaf, les MMA et une institution de prévoyance, l'Apgis. En 2009, d'autres acteurs mutualistes majeurs de l'assurance - la Macif, la Maif et la Matmut - ont fondé Sferen, une SGAM concurrente. Cette création visait 
à mettre en place une solidarité financière entre les trois mutuelles, afin de "relever en particulier les défis stratégiques de la croissance, de l'innovation, de l'optimisation des marges respectives de solvabilité et de la maîtrise des coûts", selon les termes du communiqué de presse diffusé à cette occasion, et de "permettre l'atteinte de la taille requise pour jouer le rôle majeur et actif que ces mutuelles souhaitent avoir en tant qu'acteurs mutualistes, parmi les leaders d'un marché de l'assurance en pleine mutation ", ces objectifs économiques devant être atteints "dans le respect des spécificités de chaque entité». Sferen est de fait devenu un pôle référent sur le marché de l'assurance et des services financiers.

Cependant, la Maif, forte d'un bénéfice en hausse de $11,6 \%$, a repris son indépendance en juin 2014. Rendue possible grâce aux bons résultats financiers, cette prise de distance a été motivée par un refus de "l'approche intégrative voulue par le régulateur dans la perspective de Solvabilité II». En clair, la Maif souhaite conserver son libre-arbitre dans ses choix stratégiques, qui privilégient la qualité du service plutôt que les tarifs les plus bas ${ }^{(1)}$. C'est donc en binôme, mais ouvertes à la collaboration d'autres partenaires, que Macif et Matmut travaillent à la définition du nouveau projet Sferen, qui devrait aboutir au cours du premier semestre 2015 et concernera 8,1 millions de sociétaires.

La formule SGAM est également utilisée pour concrétiser le rapprochement entre la Mutuelle générale, troisième mutuelle française, et le groupe Malakoff Médéric, fondant ainsi le premier groupe d'assurances de personnes du pays réunissant plus de 6,3 millions d'assurés et renforçant la concurrence sur le marché. Car c'est bien la loi du marché qui inspire ces nouvelles constructions à l'apparence

(1) Voir l'interview de Pascal Demurger, directeur de la Maif, mise en ligne le 22 avril 2014 sur le site Lesechos.fr (consulté le 20 octobre 2014). matriochkienne, qui n'en doivent pas moins fonctionner selon les règles de l'économie sociale. Un vrai travail d'équilibriste entre le gigantisme des chiffres, présenté comme un gage d'efficacité économique, et l'attention non quantifiable qui doit être portée au sociétaire en tant que personne, et non en tant qu'entité abstraite.

\section{La Maif se lance}

\section{dans l'économie collaborative}

En prenant une participation dans Koolicar, un site d'autopartage entre particuliers, la Maif se lance dans l'économie collaborative. Lien social, développement durable, lutte contre l'isolement et la précarité: cette économie du partage, née dans un contexte de crise sociale aiguë, offre aux acteurs historiques de l'économie sociale l'opportunité d'explorer de nouveaux modes d'expression solidaire.

\section{Les associations}

\section{Algérie: la loi contestée sur les associations sera maintenue} Le 12 janvier 2012 était adoptée en Algérie une loi sur les associations très contestée et activement combattue depuis par le milieu associatif. Ce dernier s'élève contre le renforcement du contrôle de l'Etat sur la constitution des associations et dénonce des amendements limitant la liberté d'association, tel celui autorisant l'administration à dissoudre une association pour "ingérence dans les affaires du pays». Le collectif d'associations qui s'est créé pour l'abrogation de la loi considère qu'elle "reflète dans ses dispositions une volonté manifeste de mettre au pas toute la société». Cette loi est également très critiquée à l'international, du fait des restrictions qu'elle pose aux relations entre les associations algériennes et les organisations étrangères. Ce point provoque en particulier le mécontentement de l'Union européenne, qui fait pression pour que la loi soit révisée, par la voix notamment d'Henri Malosse, 
président du Comité économique et social européen (Cese).

En juillet dernier, un projet d'amendement de la loi a été déposé au Parlement par l'Alliance Algérie verte, un groupe islamiste d'opposition à l'actuel gouvernement. Une lueur d'espoir était apparue alors que la proposition avait été acceptée par le bureau de l'Assemblée populaire nationale. Malheureusement, le gouvernement y a opposé une fin de non-recevoir. Le site Reporters.dz explique avoir eu en main un document de quatre pages signé du Premier ministre Abdelmalek Sellal, exposant "l'impossibilité juridique et pratique de cet amendement ». Son argumentation se développe essentiellement autour de la défense des prérogatives de l'Etat, au nom du maintien de l'ordre. Le dignitaire craint notamment qu'une révision de la loi ne " provoque l'anarchie dans l'organisation des réunions publiques, avec ce qu'elle peut engendrer comme troubles à l'ordre public » et dénonce la volonté du groupe AAV de "déposséder l'administration de son droit de contrôle sur les associations ». Sur ce dernier point, on peut estimer que c'est effectivement la volonté des associations de s'affranchir de la tutelle de l'Etat, mais il semble que le chemin sera encore long avant d'y parvenir.

\section{L'impact de la loi ESS sur les associations}

A la suite du vote de la loi-cadre ESS, en juillet dernier, le Mouvement associatif a fait le point sur les changements induits pour les associations et en propose un récapitulatif sur son site. Le périmètre de l'ESS tel que la loi le définit (qui inclut l'ensemble des associations) est présenté, ainsi que les futures nouvelles instances de représentation du secteur, notamment la reconnaissance du Haut Conseil à la vie associative (HCVA). Vient ensuite la description détaillée des nouvelles possibilités de financement des associations (parmi lesquelles la garantie associative, le prêt participatif social et solidaire, une meilleure attractivité des titres associatifs, le droit de possession et d'administration d'immeuble, etc.). Plusieurs mesures viennent également encourager l'engagement associatif (évaluation des congés engagement, reconnaissance des compétences des bénévoles, formation des dirigeants, etc.). Enfin, les mesures visant à simplifier le fonctionnement des associations sont présentées: obligation de publier les comptes, possibilité pour le gouvernement de procéder par ordonnance pour mettre en œuvre le " choc de simplification " administrative et, pour finir, clarification du cadre juridique des fusions entre associations.

\section{Austérité:}

\section{le lourd tribut de l'emploi associatif}

En septembre dernier, le réseau associatif Recherches et Solidarités a publié la douxième édition de son rapport annuel "La France associative en mouvement". A la suite de cette publication, le Collectif des associations citoyennes (CAC) s'alarme: en extrapolant les données du rapport, il estime qu'un emploi sur six serait menacé d'ici à 2017 dans le secteur associatif, et cela concernerait pour moitié le secteur de l'action sociale et sanitaire, dont les missions sont pourtant d'autant plus cruciales en temps de crise. Dans le cadre du plan d'austérité décrété par le gouvernement, c'est en effet de 29 milliards dont les collectivités locales devront se passer d'ici à quatre ans. Une telle contraction aura nécessairement un impact négatif sur les associations, dont $49 \%$ du budget dépend de financements publics (chiffre variable selon les secteurs et qui monte à $60 \%$ pour l'action sociale). Pour le CAC, il s'agit rien de moins que d'un immense plan social invisible à l'échelle du pays qui pourrait toucher à terme plus de 200000 emplois.

Dans un article fouillé sur la question, le site Bastamag.net, qui détaille la position du CAC, présente également l'avis plus nuancé du Mouvement associatif, au sein duquel pèsent les plus grosses associations. 
Pour ce dernier, le recul des financements aux collectivités ne se répercutera pas mécaniquement sur les associations: une telle présomption conduirait à des extrapolations exagérément alarmistes. Le mouvement reconnaît cependant que "la situation est "critique" dans le champ sanitaire et social, qui compte la moitié des salariés du secteur à but non lucratif, du fait notamment de la concurrence du privé».

Bastamag rapporte également les propos d'Hugues Sibille, qui vient de présider un comité auteur d'un rapport sur l'investissement à impact social (voir supra). Pour ce dernier, une mutation est à opérer dans les modes de financement qui verrait les associations chercher des fonds non pas auprès des pouvoirs publics, mais des investisseurs privés, par le moyen des obligations à impact social (dont les intérêts sont payés par l'Etat si les objectifs fixés ont été atteints par l'association concernée). Il s'agirait ainsi de passer de la « dépense sociale » à « l'investissement social ».

Pour le CAC, un tel changement de logique aura des conséquences désastreuses: logiques d'appels d'offres auxquels les petites structures ne sont pas équipées pour répondre, concentration, sélection des publics en fonction de leur solvabilité ou encore redéfinition des missions sur des critères de rentabilité. Alors que les difficultés financières se font sentir depuis deux ans, les structures ont d'ores et déjà mis en place différentes stratégies: hausse des prix des prestations et des cotisations, recours aux emplois précaires, quand elles ne déposent pas carrément leur bilan. Face à cette réalité déjà bien présente, le CAC dénonce " une marchandisation du secteur associatif ».

Si la fiabilité des chiffres avancés peut toujours être discutée, il est clair que les évolutions en cours visent à reporter sur le secteur privé la charge de financer l'action associative et donc pour une bonne part l'action sociale, que l'évolution en question soit présentée comme une réponse crédible à la crise ou, au contraire, comme le chemin vers la destruction progressive du tissu associatif.

\section{L'Udes et le Mouvement associatif s'accordent pour soutenir l'emploi}

L'engagement associatif a été désigné Grande Cause nationale 2014. C'est dans ce cadre que, le 25 septembre dernier, l'Union des employeurs de l'économie sociale et solidaire (Udes) et le Mouvement associatif ont signé un protocole de coopération afin de mettre en œuvre des actions pour la reconnaissance, la promotion et la défense des intérêts sociaux et économiques des associations avec pour objectif de coordonner une politique de développement de l'emploi dans ce secteur.

D’après le communiqué de presse diffusé par les deux organismes, ils s'engagent ainsi pour une durée de trois ans à travailler conjointement pour:

- "renforcer l'attractivité du secteur et favoriserle renouvellement des salariés en faisant mieux connaître les métiers des associations et leurs voies d'accès, à travers notamment la mise à disposition de guides et outils existants (guides du recrutement, de l'évolution professionnelle...), de relais d'actions de sensibilisation ou de campagnes de promotion de l'emploi associatif (portail de l'emploi dans l'économie sociale et solidaire www.emploi-ess.fr);

- développer l'emploi associatif et suivre les politiques publiques de l'emploi par:

- une coopération autour des emplois d'avenir;

- une mobilisation pour l'emploi des jeunes et le renouvellement des équipes;

- développer la qualité de l'emploi dans le secteur associatif à travers:

- le "prix pour l'emploi de qualité Mon asso s'engage!”, créé parle Mouvement associatif, en partenariat avec la mutuelle Chorum et l'Udes, afin de promouvoir et d'essaimer les meilleures pratiques de qualité de l'emploi associatif;

- la mobilisation autourde la démarchePMAE (pour "Petites et moyennes associations 
employeuses »), développée afin de renforcer les compétences des accompagnateurs des petites et moyennes associations employeuses pour un emploi associatif de qualité;

- l'encouragement des pratiques de mutualisation et des logiques de parcours d'emplois associatifs;

- la formation des bénévoles.»

\section{Le guide 2014 pour l'égalité femmes- hommes dans les associations}

La direction départementale de la cohésion sociale du Rhône a élaboré l'édition 2014 de son Guide pour l'égalité entre les femmes et les hommes dans les associations, téléchargeable sur son site. Ce guide, édité par le ministère des Droits des femmes, de la Ville, de la Jeunesse et des Sports, a pour objet « de fournir aux acteurs associatifs un référentiel de diagnostic et des éléments opérationnels pour améliorer, y compris dans les organes dirigeants, l'égalité entre les femmes et les hommes. Il est en concordance avec la loi du 4 août 2014 ».

\section{Les propositions du Collectif des associations citoyennes pour simplifier la vie associative}

Dans le cadre du "choc de simplification" et alors que la loi ESS nouvellement votée autorise le gouvernement à procéder par ordonnance pour simplifier les procédures administratives concernant les associations et les fondations et que le député Yves Blein s'est vu confier une mission pour "travailler sur tous les leviers de simplification à chaque étape de la vie d'une association ", le Collectif des associations citoyennes (CAC) a fait les propositions suivantes:

- simplifier les dossiers des demandes de subvention;

- proportionner les contrôles à la réalité de la vie associative (en abandonnant des exigences inspirées des règles européennes pour des dossiers qui n'en relèvent pas); - réhabiliter les subventions de fonctionnement centrées sur le projet associatif; - financer les projets sur la base d'un montant déterminé et non d'un pourcentage des dépenses, aller vers des avances automatiques, constituer un fonds d'urgence à taux zéro pour répondre aux difficultés de trésorerie les plus importantes, notamment lorsque celles-ci sont la conséquence directe des délais de prise de décision des collectivités publiques;

- assouplir la règle de l'antériorité en autorisant les associations à commencer la réalisation des projets ou à assurer la continuité des actions, ce qui signifie que les dépenses correspondantes sont éligibles même si elles précèdent la décision formelle d'attribution des crédits;

- aller vers des méthodes d'évaluation qualitatives adaptées;

- créer des lieux de dialogue permanents au niveau de l'Etat ou localement, au niveau des collectivités, pour éviter la résurgence des complexités.

LA RÉDACTION 